\title{
O cuidar do enfermeiro especialista em reabilitação físico-motora*
}

\author{
THE CARE OF THE NURSE SPECIALIZED IN PHYSICAL-MOTOR REHABILITATION \\ EL CUIDAR DEL ENFERMERO ESPECIALISTA EN REHABILITACIÓN FÍSICO-MOTORA
}

\author{
Valéria Barreto Esteves Leite ${ }^{1}$, Ana Cristina Mancussi e Faro²
}

\section{RESUMO}

O processo de reabilitação físicomotora tem por objetivo principal a adaptação das pessoas com deficiência física às novas condições de vida. Este trabalho tem por objetivo descrever as atividades desenvolvidas pelo enfermeiro de reabilitação em uma unidade de internação de pacientes em reabilitação físico-motora e destacar sua importância frente à equipe de reabilitação. Dentre as principais atividades desenvolvidas destacamos a manutenção das necessidades fisiológicas, onde a equipe de Enfermagem executa, orienta, demonstra, supervisiona e reavalia as condições de autocuidado destes pacientes. O enfermeiro atua, também, na prevenção de possíveis complicações que podem ocorrer frente à suscetibilidade peculiar desta clientela.

\section{DESCRITORES}

Enfermagem em reabilitação.

Pessoas portadoras de

deficiência.

Atividades cotidianas.

\begin{abstract}
The main objective of the physical-motor rehabilitation process is to help people with physical deficiency adapt to their new condition of life. This paper aims to describe the activities developed by the rehabilitation nurse in a unit for patients undergoing physicalmotor rehabilitation and to detail his/her importance for the rehabilitation team. Among the most important activities carried out are the maintenance of the patient's physiological needs, which the nursing staff executes, orients, demonstrates and supervises, and the reevaluation of the conditions of such patients to care for themselves. The nurse also acts in the prevention of possible complications that could occur given the unique susceptibility of this clientele.
\end{abstract}

\section{KEYWORDS}

Rehabilitation nursing.

Disabled persons.

Activities of daily living.

\section{RESUMEN}

El processo de rehabilitación físico-motora tiene como principal objetivo la adaptación de las personas con deficiencia física a las nuevas condiciones de vida. Este trabajo tiene por objetivo describir las actividades desarrolladas por el enfermero de rehabilitación físico-motora y destacar su importancia frente al equipo de rehabilitación. Dentro de las principales actividades desarrolladas, se destaca la manutención de las necesidades fisiológicas, donde el equipo de enfermería ejecuta, orienta, demuestra, supervisa y reevalúa las condiciones de auto cuidado de estos pacientes. El enfermero también actúa en la prevención de pisibles complicaciones que pueden ocurrir frente a la susceptibilidad peculiar de esta clientela.

\section{DESCRIPTORES}

Enfermería en rehabilitación. Personas con discapacidades. Actividades cotidianas.

\footnotetext{
* Trabalho realizado durante o I Curso de Especialização em Enfermagem em Reabilitação da Escola de Enfermagem da USP (EEUSP), 2002.

1 Enfermeira. Especialista em Reabilitação. graox@zaz.com.br

2 Livre Docente na área Enfermagem em Reabilitação do Departamento de Enfermagem MédicoCirúrgica da EEUSP. Coordenadora do Curso de Especialização em Enfermagem em Reabilitação. rafacris@usp.br
} 


\section{INTRODUÇÃO}

Partindo do princípio de que o cuidar do corpo humano exige, necessariamente, um olhar para a dimensão total do ser, inclusive de sua essência existencial, torna-se imprescindível, para nós enfermeiros, uma maior conscientização acerca do importante papel que desempenhamos ao interferir no espaço de privacidade das pessoas dependentes de nossas intervenções, como aqueles que apresentam deficiência física ${ }^{(1)}$.

O enfermeiro por sua formação e atuação profissional desenvolve papéis nos âmbitos educativo, gerencial, na coordenação e implementação da assistência de Enfermagem ao binômio paciente e família e à comunidade.

O papel educativo do enfermeiro foi considerado desde o início da Enfermagem Moderna e hoje os hospitais, como instituições de saúde, têm ampliado a sua finalidade curativa para incluir, também os papéis de prevenção e reabilitação ${ }^{(2)}$.

O enfermeiro tem características que facilitam o seu papel educador com o paciente: é ele o elemento da equipe que mais tempo permanece ao lado do paciente e tem a capacidade de observá-lo e considerálo como todo e não apenas como um caso $^{(3)}$.

A Reabilitação é um processo dinâmico, orientado para a saúde, que auxilia um indivíduo que está enfermo ou incapacitado para atingir seu maior nível possível de funcionamento físico, mental, espiritual, social e econômico. O processo de Reabilitação ajuda a pessoa a atingir uma aceitável qualidade de vida com dignidade, auto-estima e independência ${ }^{(4)}$.

A reabilitação faz parte dos cuidados de Enfermagem enquanto um modelo assistencial, bem como uma especialidade. Os esforços da reabilitação devem começar no contato inicial com o paciente. Os princípios de reabilitação são básicos para o cuidado, mesmo na ausência da deficiência física e suas incapacidades, mas considerando o modelo assistencial da reabilitação, essencialmente preventivo, educativo e que aborda o binômio paciente/cuidador familiar.

A reabilitação dos portadores de lesão medular, por exemplo, se inicia na fase aguda, logo após a ocorrência do trauma, principalmente através dos cuidados preventivos de formação de úlceras de decúbito, deformidades dos segmentos paralisados, esvaziamento vesical e intestinal realizados de maneira adequada e cuidados com os distúrbios vasomotores ${ }^{(5)}$.

A ênfase da Reabilitação está voltada para a restauração da independência do paciente ou recuperação do seu nível de função pré-enfermidade ou pré incapacidade no menor tempo possível.

Neste trabalho, é nosso objetivo destacar e descrever as principais atividades desenvolvidas pelo enfermeiro em uma unidade de internação de Reabilitação de uma instituição filantrópica no município de São Paulo.

Trata-se de um relato de experiência profissional o qual contempla descrição de procedimentos com estratégias de intervenção, de interesse para a atuação de enfermeiros na Reabilitação na saúde do adulto com lesão medular.

A referida instituição desenvolve um trabalho sem fins lucrativos no sentido de tratar as pessoas com deficiências físicas de todo o Brasil e auxiliar na reintegração à sociedade.

Em estudo realizado pelo setor de Psicologia desta instituição, observou que o retorno dos pacientes às atividades sociais e profissionais está diretamente relacionado com a condição social de vida de cada um deles e o apoio familiar ou não que cada um recebe de seus entes mais próximos. Há a presença também de algumas barreiras arquitetônicas e físicas de responsabilidade governamental que dificultam o retorno de pacientes com deficiência física à sociedade.

Isto posto, passaremos à descrição da atuação do Enfermeiro especialista em Reabilitação junto aos pacientes internados nesta instituição.

\section{Atuação do Enfermeiro}

Para que estes pacientes possam ser admitidos no programa, é imprescindível que estejam clinicamente estáveis o que possibilita a internação na unidade de reabilitação, pois o objetivo é a adaptação destes à nova situação de vida, imposta por sequelas da patologia limita-dora de suas funções motoras.

A equipe de Reabilitação é constituída por Médico Fisiatra, Enfermeiro, Fisioterapeuta, Terapeuta Ocupacional, Fonoaudiólogo, Psicológo e Assistente Social. Acompanham de perto a reabilitação, os profissionais Orto-dontistas/Protético, Engenheiro de reabilitação (responsáveis pela confecção de órteses e equipamentos que visam auxiliar em posicionamento e atividades destes pacientes). 
Valéria B. Esteves Leite Ana Cristina M. e Faro
O paciente é o elemento chave da equipe de reabilitação. Ele é o foco do esforço da equipe e aquele que determina os resultados finais do processo $^{(4)}$. A família é incorporada à equipe e é reconhecida como um sistema dinâmico que participa como um apoio contínuo, na solução de problemas e aprende a realizar cuidados contínuos necessários.

A assistência de Enfermagem na reabilitação tem como principais objetivos auxiliar o paciente a se tornar independente o máximo que puder dentro de suas condições, promover e incentivar o autocuidado através de orientações e treinamento de situações, preparar o deficiente físico para uma vida social, familiar da melhor maneira possível e com qualidade.

Autocuidado é a prática de atividades, iniciadas e executadas pelos indivíduos, em seu próprio benefício, para a manutenção da vida, da saúde e do bem-estar. As capacidades do indivíduo para engajar-se no autocuidado acham-se condicionadas pela idade, estado de desenvolvimento, experiência de vida, orientação sóciocultural, saúde e recursos disponíveis. Normalmente, pessoas adultas cuidam voluntariamente de si mesmas. Bebês, crianças, pessoas idosas, pessoas doentes e pessoas incapacitadas requerem total assistência ou prestação de cuidados, com atividades de autocuidado ${ }^{(6)}$.

As necessidades dos pacientes são observadas por toda a equipe de reabilitação para que as ações sejam direcionadas a cada um e também para se ter parâmetro para o dimensionamento de pessoal, considerando o tempo gasto para sua manutenção básica o que, conforme o caso, pode exigir por volta de 10 horas diárias de Enfermagem, a quantidade e a qualidade de assistência exigida.

Os principais problemas e intervenções de Enfermagem no âmbito da reabilitação de deficientes físicos, durante a internação, nesta instituição, se referem à manutenção da integridade física e motora. Conseqüentemente à diminuição ou ausência da mobilidade e sensibilidade, cuidados específicos e de caráter preventivo, são relevantes ao bem estar do paciente.

Assim, implementamos um programa preventivo e efetivo de mudança de decúbito e posicionamento no leito e na cadeira de rodas, com ênfase para evitar deformidades e/ou úlceras por pressão, sobretudo em membros inferiores e nas regiões mais comuns como a occiptal, sacra, cotovelos, joelhos e calcâneos. Destacamos, neste pro- grama, a periodicidade para a mudança de decúbito que varia entre 2 a 4 horas, bem como os posicionamentos adequados às condições do paciente.

Os pacientes são orientados, durante a internação, sobre a importância do autocuidado, sobretudo quanto à higiene corporal, alimentação e hidratação, estabelecendo conjuntamente metas dentro das possibilidades do momento.

Com o enfoque na realização das atividades da vida diária (AVD) da maneira mais independente possível, torna-se necessário que o enfermeiro reabilitador conheça os hábitos e o estilo de vida do paciente no contexto da família e da sociedade. Esta atuação compreende o autocuidado para a capacidade de vestir/despir, alimentar-se, fazer higiene pessoal e íntima, prevenir deformidades de articulação, complicações respiratórias e vasculares.

Destacamos o autocuidado voltado à reeducação das eliminações vesicais e intestinais segundo condições funcionais, físico-motoras, sociais e familiares. São estabelecidos, face a tais considerações, programas de treinamento ao binômio paciente-família (cuidador familiar) conforme proposto por especialistas na área ${ }^{(7-13)}$. Outras orientações pertinentes à esfera biopsico-espiritual são direcionadas à família e ao paciente, estimulando a participação nos cuidados, reconhecendo os limites e as possibilidades de cada um.

A cada nova internação, o enfermeiro executa o Processo de Enfermagem que é constituído de exame físico e entrevista, sendo que a partir daí é realizado o levantamento de dados específicos relacionados à deficiência física e incapacidades que nortearão as ações da Enfermagem especializada para a assistência à pessoa com defíciência física.

Durante a entrevista são verificados os hábitos de vida relacionados à alimentação, hidratação, eliminação urinária e intestinal, bem como o padrão atual dessas últimas.

Outros dados específicos são identificados, os quais são relacionados à avaliação uro-dinâmica, conhecimento e realização de manobras de esvaziamento vesical, intestinal, cateterismo vesical intermitente - técnica limpa, medicamentos utilizados no momento e habilidades físicomotoras voltadas à manutenção destes hábitos $\mathrm{e}$ atreladas às AVDs, vestuário, transferências, posicionamentos no leito e em cadeira de rodas, além do uso de órteses e verificação da adequação das mesmas. 
Por meio do exame físico, observa-se também as condições gerais de pele e anexos, presença ou não de deformidades.

A avaliação de Enfermagem necessita de uma abordagem holística. O estado físico, mental, emocional, espiritual, social e econômico são considerados.

A partir do histórico de Enfermagem, se estabelecem os problemas levantados e realiza-se a Prescrição de Enfermagem para nortear as ações da equipe de Enfermagem. Através das anotações estamos em constante acompanhamento com o desenvolvimento dos pacientes.

É importante que sejam criadas condições favoráveis à promoção de independência ao paciente. Deve haver percepção pelo enfermeiro reabilitador em saber o que o paciente tem condições de realizar sozinho, mesmo que com dificuldade, e aquilo que há necessidade de auxílio.

Normalmente, o dia a dia dos pacientes em Reabilitação é intenso. As atividades de fisioterapia, psicologia, arteterapia, hidroterapia, natação, e outras ocupam o paciente por longos períodos de tempo durante o dia. É necessário uma estreita relação entre a Enfermagem e as demais equipes integrantes do programa para que as atividades possam ser realizadas de maneira adequada ao tratamento de reabilitação do paciente.

O enfermeiro especializado em reabilitação é responsável, como todo profissional enfermeiro, pela supervisão e orientação a toda equipe de Enfermagem em Reabilitação; pela realização da escala de folgas e dimensionamento de pessoal; supervisão e controle sobre o Processo de Enfermagem e "Primary Nurse", para que se cumpra adequadamente conforme princípios da instituição; realização de avaliação holística do paciente e acompanhamento de seu desenvolvimento na reabilitação; realização de relacionamento terapêutico; pela integração dos funcionários entre si e com os pacientes; intermediador da Enfermagem com os demais componentes da Equipe de Reabilitação (fisioterapeuta, fono-audiólogo, terapeuta ocupacional, e outros); planejamento de reuniões de treinamento e aprimoramento dos funcionários que atuam com Reabilitação; atuação em conjunto com a Educação Continuada para melhoria da assistência prestada; treinamento e avaliação do funcionário admitido na Equipe de Reabilitação; realização do Processo de Enfermagem; avaliação e acompanhamento do paciente no que diz respeito à sua evolução na reabilitação; orientações quanto aos cuidados com alimentação, higiene, eliminações; auto- cuidado à família e acompanhantes; realização de curativos específicos que requerem atenção especial, como feridas infectadas, por exemplo.

Uma vez que se tem como princípios de que reabilitação é um processo com começo, meio e fim, a alta hospitalar não pode ser encarada como um evento mas sim como um processo. As orientações de alta são realizadas durante toda a internação e não somente no dia da alta hospitalar.

O enfermeiro é de fundamental importância na equipe designada para a realização da visita domiciliária pré e pós alta para que as adaptações no ambiente sejam orientadas de maneira adequada aos cuidados em casa.

É de extrema importância que o paciente/família seja informado com antecedência ao momento de sua alta para que todas as dúvidas sejam realmente esclarecidas e que sintam segurança e, principalmente, confiança em si mesmos para ir para casa com sua motivação principal presente: a independência.

\section{CONSIDERAÇÕES FINAIS}

A reabilitação física-motora não pode ser entendida como uma complementação ao tratamento do deficiente físico. Trata-se de um processo de cuidar precoce, abrangente, holístico enquanto um modelo assistencial, essencialmente educativo.

Por meio da Sistematização da Assistência, o enfermeiro reabilitador desenvolve um Plano de Cuidados destinados a facilitar a reabilitação, restaurar e manter níveis saudáveis de vida e evitar complicações.

O enfermeiro reabilitador desenvolve um papel fundamental de Educador, bem como de Implementador de cuidados, Conselheiro e Consultor, muitas vezes o responsável pelo planejamento geral de reabilitação.

Cabe ressaltar aqui que o enfermeiro atua como líder de equipe, colocando que as estruturas organizacionais tradicionais, caracterizadas por formas autoritárias nas quais os trabalhadores eram dirigidos ou instruídos sobre o que, como e quando fazer, estão dando passagem a formas democráticas de organização, caracterizadas por um estilo participativo e cooperativo ${ }^{(14)}$.

É relevante salientar que o enfermeiro enquanto líder deve conhecer a si mesmo e que isso pode ser facilitado com a utilização de quatro preceitos: 1. você é seu melhor professor; 2 . aceite sua res- 
Valéria B. Esteves Leite Ana Cristina M. e Faro ponsabilidade; 3 . você pode aprender o que quiser; 4 . o verdadeiro entendimento vem da reflexão sobre a experiência pessoal ${ }^{(14)}$.

No tocante à especialidade e especificidade do processo de cuidar das diversas patologias neuromúsculo-esqueléticas congênitas, adquiridas, traumáticas ou não, requer conhecimento e habilidades técnicas e atitudinais por parte do enfermeiro, compondo e implementando um "saber cuidar" com métodos e terapêuticas próprias de um especialista.

\section{REFERÊNCIAS}

(1) Machado WCA. O papel do enfermeiro no cuidar de clientes portadores de deficiência. [online]. São Paulo: Entre Amigos - Rede de Informações sobre Deficiência; [s.d.]. Disponível em: <http://www. entreamigos.com.br/textos/reabili/opapel.html> (19 jan. 2003)

(2) Santos BRL, Mendes EEM. Programa de assistência de enfermagem a clientes portadores de danos cardiovasculares, no ambulatório de um hospital de ensino de Porto Alegre. Rev Gaúcha de Enferm 1983; 4(1):61-73.

(3) Zago MMF. O papel do enfermeiro hospitalar no ensino do paciente. Rev Esc Enferm USP 1992; 26(3):359-64.

(4) Brunner LS, Suddarth DS. Princípios e práticas de reabilitação. In: Smeltzer SC, Bare BG. Brunner \& Suddart: tratado de enfermagem médico-cirúrgica. Rio de Janeiro: Guanabara Koogan; 1993. p. $181-207$.

(5) Greve JMDA. Fundamentos clínicos do tratamento de reabilitação na lesão medular. Âmbito Hosp 1994; 6(67):55-64.

(6) George JB. Teorias de enfermagem: fundamentos para a prática profissional. Porto Alegre: Artes Médicas; 1993.

(7) Madureira NCM. O saber-fazer do cuidador familiar da pessoa com defíciência física: um estudo no pré e trans-reabilitação. [dissertação] São Paulo (SP): Escola de Enfermagem da USP; 2000.
(8) Moróoka M. Autocateterismo vesical intermitente técnica limpa: descrição do procedimento realizados pelos pacientes com lesão medular. [dissertação] São Paulo (SP): Escola de Enfermagem da USP; 2000.

(9) Faro ACM e. Do diagnóstico à conduta de enfermagem: a trajetória do cuidar na reabilitação do lesado medular. [tese] São Paulo (SP): Escola de Enfermagem da USP; 1995.

(10) Faro ACM e. Assistência ao paciente com traumatismo raquimedular. In: Ventura M, Faro ACM, Onoe EK, Utimura M. Enfermagem ortopédica. São Paulo: Ícone; 1996. p. 175-89.

(11) Faro ACM e. Cuidar do lesado medular em casa: a vivência singular do cuidador familiar. [livre docência] São Paulo (SP): Escola de Enfermagem da USP; 1999.

(12) Mancussi AC. Assistência ao binômio paciente/ família na situação de lesão traumática da medula espinhal. Rev Lat Am Enferm 1998; 6(4):67-73.

(13) Tonello AS. Aspectos de reeducação intestinal em lesados medulares. [dissertação] São Paulo (SP): Escola Paulista de Medicina, Universidade Federal de São Paulo; 1999.

(14) Galvão CM, Trevisan MA, Sawada NO. A liderança do enfermeiro no século XXI: algumas considerações. Rev Esc Enferm USP 1998; 32(4): 302-6.
Correspondência: Ana Cristina M. e Faro Depto. Enfermagem Médico-Cirúrgica da EEUSP Av. Dr. Enéas de Carvalho Aguiar, 419 - Cerqueira César - São Paulo CEP - 05403-000 - SP

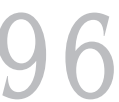

Rev Esc Enferm USP $2005 ; 39(1): 92-6$ 